LIVER

\title{
Cholesterol synthesis inhibition distal to squalene upregulates biliary phospholipid secretion and counteracts cholelithiasis in the genetically prone C57L/J mouse
}

\author{
G A Clarke, G Bouchard, B Paigen, M C Carey
}

\begin{abstract}
Background and aims: Newly synthesised cholesterol contributes poorly to biliary lipid secretion but may assume greater importance when the rate limiting enzyme 3-hydroxy-3-methylglutaryl-CoA reductase (HMGR) is upregulated. As this occurs in the gall stone susceptible C57L/J inbred mouse, we employed two cholesterol biosynthesis inhibitors, Tu 2208 and Ro 48-8071, potent inhibitors of squalene epoxidase and oxidosqualene-lanosterol cyclase, respectively, to assess their potential in preventing cholesterol cholelithiasis in the C57L/J mouse strain. Mice were fed a lithogenic diet comprising a balanced nutrient intake with $15 \%$ dairy fat, $1 \%$ cholesterol, and $0.5 \%$ cholic acid added. Methods: We determined gall stone phenotype, HMGR activity, biliary lipid secretion rates, and counterregulatory events in male C57L/J mice and gall stone resistant AKR treated with Tu 2208 (30$60 \mathrm{mg} / \mathrm{kg} /$ day) or Ro $48-8071$ (30-100 mg/kg/day), while ingesting chow or the lithogenic diet. Results: Both agents reduced the gall stone prevalence rate from $73 \%$ to $17 \%$ in C57L/J mice, inhibited HMGR activity, and decreased hepatic cholesterol concentrations without appreciably influencing biliary cholesterol secretion. In C57L as well as AKR mice, both agents increased biliary phospholipid (which is mostly phosphatidylcholine) secretion rates and at the highest doses effectively reduced the biliary cholesterol saturation index.

Conclusions: Cholesterol biosynthesis inhibitors acting distally to squalene do not reduce biliary cholesterol secretion rates despite reductions in cholesterol biosynthesis and hepatocellular levels. However, they effectively prevent gall stone formation through stimulation of pathways that lead to enhanced biliary phospholipid secretion.
\end{abstract}

See end of article for authors' affiliations

Correspondence to:

Dr M C Carey, Department of Medicine, Thorn 1430 Brigham and Women's Hospital, 75 Francis Street, Boston, MA 02115, USA; mccarey@rics.bwh.

harvard.edu Accepted for publication 26 August 2003

D novo biosynthesis, plasma lipoproteins, and dietary intake plus resorbed biliary cholesterol as chylomicron remnants, comprise the sources of hepatic cholesterol molecules for biliary secretion. ${ }^{1}$ Cholesterol that is taken up or newly synthesised by the hepatocyte may be secreted directly into bile, esterified by acyl coenzyme A: cholesterol O-acyltransferase $2,{ }^{2}$ or used as substrate for bile salt synthesis. ${ }^{3}$ The preponderance of current evidence points towards diet as the principal source of biliary cholesterol secretion in rodents and endogenous lipoprotein cholesterol as the predominant substrate for bile salt synthesis. ${ }^{4-6}$ Because of the complexity of hepatic metabolism of cholesterol and the consequent difficulties in its quantitation, the respective contributions of each cholesterol source to biliary secretion remain unsettled. Moreover, these contributions may differ between laboratory animals and humans and between basal and cholesterol hypersecretory-that is, lithogenic states.

In previous work, we demonstrated relative hypersecretion of biliary cholesterol compared with the other biliary lipids in the gall stone susceptible C57L/J mouse. ${ }^{7}$ Accordingly, when fed a lithogenic diet high in cholesterol and cholic acid, C57L/J mice exhibit an approximately $80 \%$ gall stone prevalence rate. ${ }^{8}$ Moreover, compared with gall stone resistant mouse strains such as AKR/J, C57L/J mice exhibit an apparent increase in hepatic uptake of high density lipoprotein cholesterol, ${ }^{9}$ augmentation of intracellular cholesterol transport, ${ }^{10}$ and failure to downregulate cholesterol biosynthesis. ${ }^{11}$ All of these factors could promote biliary cholesterol hypersecretion and lithogenicity. Despite the theoretical effect of reducing de novo cholesterol synthesis, which might affect biliary cholesterol secretion by depleting hepatic cholesterol stores, ${ }^{12-15}$ it has been impossible hithertofore to test the physiological impact of inhibiting cholesterol biosynthesis in mice. This is occasioned because rodents respond to "statins", the benchmark class of cholesterol synthesis inhibitors, in a paradoxical manner by increasing transcription of 3-hydroxy-3-methylglutaryl-CoA reductase (HMGR) to such an extent that overall cholesterol biosynthesis is actually increased. ${ }^{16}{ }^{17}$ In fact, in hamsters, statins may induce lithogenic bile and gall stones. ${ }^{18}$ Furthermore, statins also inhibit formation of isoprenoid precursors, which are important products of the cholesterol synthesis pathway with protean physiological roles (fig 1).

For these reasons we explored Tu 2208 (Yoshitomi Pharmaceuticals Ltd, Osaka, Japan), an inhibitor of squalene epoxidase (fig 1), as inferred from the properties of its antecedent analogue, $\mathrm{Tu} 2708,{ }^{19}$ and Ro 48-8071 (F Hoffmann-La Roche Ltd, Basel, Switzerland), an inhibitor of oxidosqualene-lanosterol cyclase, ${ }^{15}$ shown to effectively suppress de novo cholesterol synthesis. In contrast with the statins, these agents inhibit enzymes that act on substrates formed distally to the synthesis of isoprenoid precursors (fig 1). By administering these cholesterol synthesis inhibitors

\footnotetext{
Abbreviations: LD, lithogenic diet; Mdr2, multidrug resistance protein, isoform 2 (where italicised in lowercase indicates the corresponding murine gene); bsep, bile salt export protein; ABC, ATP binding cassette (class and number are suffixed for official designations - that is, B4 and B 11 for $m d r 2$ and bsep, respectively); Lith, murine cholesterol gall stone alleles; HMGR, 3-hydroxy-3-methylglutaryl-CoA reductase; SREBP, sterol regulatory element binding proteins; PC, phosphatidylcholine (biliary phospholipid); CSI, cholesterol saturation index
} 


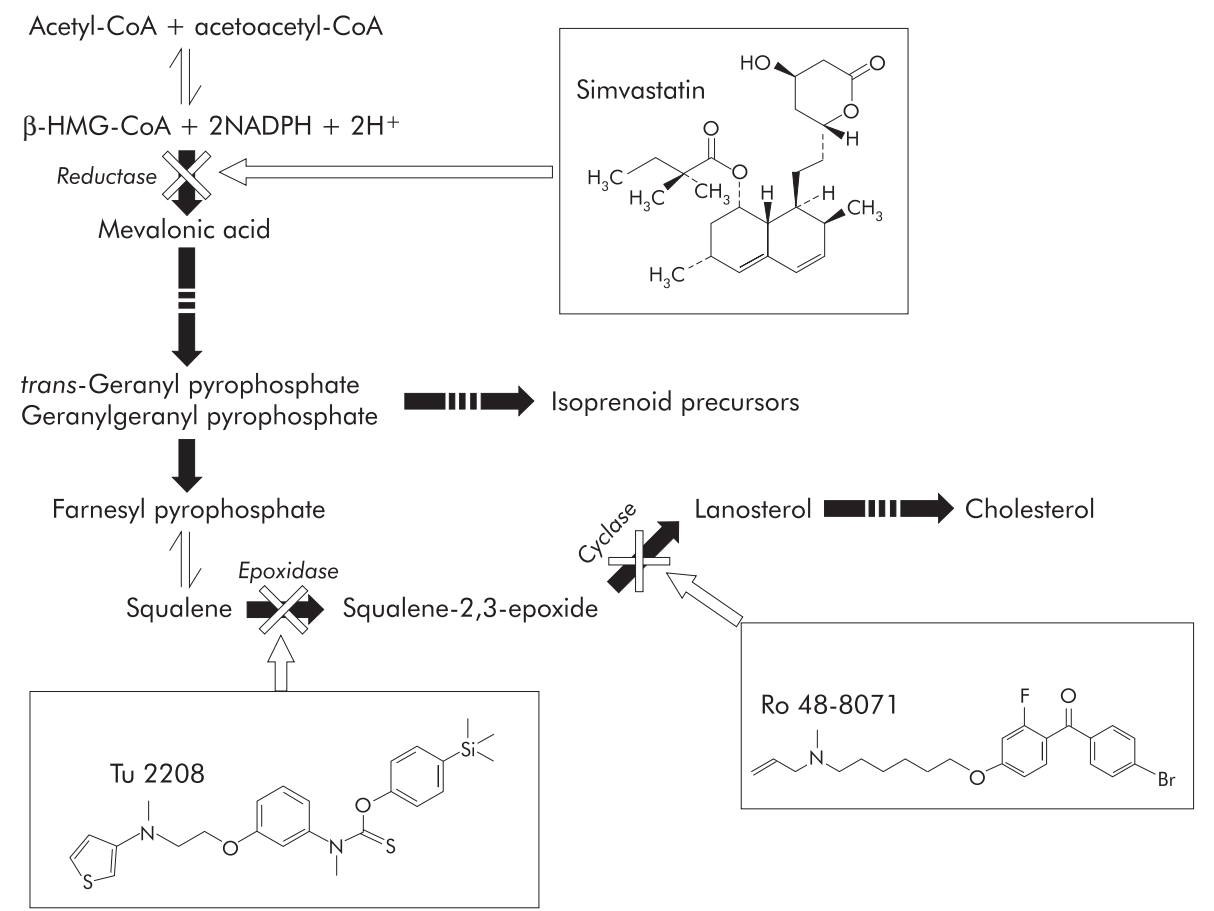

Figure 1 Flow chart summarising the cholesterol biosynthetic pathway, together with the structures and sites of action of the Tu 2208 and Ro 48-8071 inhibitors employed in this study. Black arrows indicate an irreversible step, broken arrows indicate that one or more intermediate steps have been omitted for clarity. Solid arrows with an overlying open X indicate the site of each inhibitor's action. Standard symbols are used to indicate reversible steps.

to $\mathrm{C} 57 \mathrm{~L}$ gall stone prone mice, we tested the hypotheses that decreasing de novo biosynthesised cholesterol would influence biliary lithogenicity and cholesterol gall stone prevalence rates. We found that neither agent reduced biliary cholesterol secretion despite depletion of hepatic cholesterol stores and inhibition of HMGR. None the less, both agents caused bile to become desaturated with cholesterol and prevented gall stone formation, principally by increasing biliary phospholipid secretion rates. These findings indicate that cholesterol biosynthesis inhibitors acting distally to HMGR can prevent cholesterol gall stone formation but this effect is achieved by upregulating biliary phospholipid secretion most likely through counterregulatory mechanisms.

\section{MATERIALS AND METHODS \\ Materials}

$\mathrm{Tu} 2208$ was a gift of $\mathrm{Dr} \mathrm{T}$ Yamauchi (Yoshitomi Pharmaceuticals) and Ro 48-8071 was a gift of Dr Olivier Morand (F Hoffmann-La Roche, Ltd). Pure crystalline simvastatin was received from Merck, Sharp and Dohme (Ireland, Ltd). Protein dye binding substrates and standards were obtained from Biorad Inc. (Hercules, California, USA). Precast polyacrylamide gels, sample, running and transfer buffers were obtained from Novex-Invitrogen (Carlsbad, California, USA). The primary monoclonal antibody, MC204, recognising murine multidrug resistance protein, isoform 2 (Mdr2), was obtained from Kamiya Biomedical Company (Seattle, Washington, USA). A polyclonal antibody raised against the $\mathrm{C}$ terminus of rat bile salt export protein (bsep) was a generous gift of Dr Bruno Stieger (University Hospital, Zurich, Switzerland). ${ }^{20}$ Antibodies were detected using appropriate horseradish peroxidase conjugated secondary antibodies (Jackson Immunoresearch Laboratories, West Grove, Pennsylvania, USA) with a chemiluminescence kit (New England Nuclear Inc., Boston, Massachusetts, USA). A
"Quantity One" densitometer (Biorad Inc.) was used to measure expression density.

\section{Inbred mice}

Six week old male C57L and AKR mice were supplied by the Jackson Laboratory (Bar Harbor, Maine, USA). They were acclimatised for two weeks in the animal research facility of Harvard Medical School and maintained under normal 12 hour alternating light-dark conditions, with water available ad libitum. All experiments were executed according to the accepted criteria for the care and experimental use of laboratory animals, and euthanasia was consistent with recommendations of the American Veterinary Medical Association. All protocols were approved by the Institutional Animal Care and Use Committee of Harvard University.

\section{Drug administration and dosage}

Previously, we had quantified the daily weight consumption of the lithogenic diet (LD) (prepared in the diet kitchen of the Jackson Laboratory) by C57L and AKR inbred mouse strains ${ }^{21}$ and thereby calculated the concentrations of drug necessary to compound with the daily feeds. Chow and LDs (plus drug at each dosage level) were compounded in the diet kitchen of the Jackson Laboratory. Eight week old male C57L mice were fed LD plus Tu $220830 \mathrm{mg} / \mathrm{kg} /$ day (group Tu 30, $\mathrm{n}=14$ ) and $60 \mathrm{mg} / \mathrm{kg} /$ day (group Tu 60, n=6), Ro 48-8071 $30 \mathrm{mg} / \mathrm{kg} /$ day (group Ro 30, $\mathrm{n}=15$ ), $60 \mathrm{mg} / \mathrm{kg} /$ day (group Ro 60, $\mathrm{n}=6$ ), and $100 \mathrm{mg} / \mathrm{kg} /$ day (group Ro 100, $\mathrm{n}=6$ ). Contemporaneous controls $(n=12)$ were fed LD alone or LD plus simvastatin $10 \mathrm{mg} / \mathrm{kg} /$ day. AKR mice were fed LD alone $(\mathrm{n}=11)$ or $\mathrm{LD}$ plus simvastatin $(n=5)$. While on diet, animals were sacrificed at 56 days using pentobarbital sodium $4.5 \mathrm{mg} /$ $100 \mathrm{~g}$ body weight for anaesthesia (Nembutal; Abbott Laboratories, Chicago, Illinois, USA). 


\section{Gall bladder phenotypes, and collection of hepatic bile and blood}

To determine gall stone prevalence, an upper midline laparotomy was performed following an overnight fast, the cystic duct was ligated and transected, and a cholecystectomy performed. The liver was removed immediately, weighed, snap frozen in liquid $\mathrm{N}_{2}$, and stored at $-70^{\circ} \mathrm{C}$. Gall bladder volume was determined by water displacement, ${ }^{21}$ and polarising microscopy was performed on bile that was digitally expressed from the perforated fundus of each gall bladder $(n=5-15)$ to determine the presence of mature stones, sandy stones, crystals, liquid crystals, and mucin gel, as described by Wang and colleagues. ${ }^{8}$ Blood was aspirated from the left ventricle, plasma prepared, and stored at $-70^{\circ} \mathrm{C}$.

\section{Biliary lipid secretion}

In a separate study, eight week old male C57L mice were fed $\mathrm{LD}$ plus $\mathrm{Tu} 60 \mathrm{mg} / \mathrm{kg} /$ day (group Tu 60), Ro 30, 60, or $100 \mathrm{mg} / \mathrm{kg} /$ day (group Ro 30, 60, or 100, respectively), or LD plus simvastatin $10 \mathrm{mg} / \mathrm{kg} /$ day (group simva); control animals (given no drug) were fed LD alone. To obviate the possibility that observed changes in biliary lipid secretion might be idiosyncratic to mutant murine cholesterol gall stone alleles (Lith alleles), AKR controls were also employed and fed LD alone, LD plus simvastatin $10 \mathrm{mg} / \mathrm{kg} /$ day, and LD plus Ro $100 \mathrm{mg} / \mathrm{kg} /$ day. Mice were anaesthetised as described above, after 14 days on diet. The cystic and distal common bile ducts were ligated after midline laparotomy. An oblique cut was made in the mid common bile duct using a microscissors, permitting cannulation with a polyethylene catheter of $0.61 \mathrm{~mm}$ external diameter (Beckton Dickinson Inc., Sparks, Maryland, USA), which was then sutured in position. Hepatic bile was collected for one hour into a tared container, with the animal's temperature maintained at $37^{\circ} \mathrm{C}$ under a heating lamp. Six animals were included in each group but in a small number, bile duct cannulation was not possible and hence the adjusted $\mathrm{n}$ values are detailed in the results section.

\section{Analyses}

Total plasma cholesterol was measured using standard enzymatic techniques. ${ }^{22}$ Biliary phospholipid compositions were determined using the Bartlett assay. ${ }^{23}$ We verified that the Bartlett assay was reporting phospholipid as opposed to other phosphorylated compounds by positively correlating its results with a commercially available choline assay (Wako Chemicals USA, Inc., Richmond, Virginia, USA). Bile salts were assayed using the $3 \alpha$-hydroxysteroid dehydrogenase assay, ${ }^{24}$ and biliary cholesterol, as well as bile salt molecular species, by high performance liquid chromatography. ${ }^{25}$ Cholesterol saturation indices (CSIs) were determined using critical table ${ }^{26}$ derived for taurocholate, the principal murine bile salt on the LD. As it has been shown that HMGR activity exhibits a positive correlation with the absolute rate of hepatic cholesterol biosynthesis, as measured by incorporation of ${ }^{14} \mathrm{C}$-octanoate into sterols, ${ }^{27}$ we assayed HMGR activity ex vivo in the presence of ${ }^{14} \mathrm{C}$-HMG-CoA, glucose 6phosphate, NADP, and glucose 6-phosphatase using hepatic microsome fractions of standardised protein concentration according to the methods described by Doerner and colleagues. ${ }^{27}$ Protein concentrations were determined using the Bradford assay. ${ }^{28}$ Following homogenisation and lipid extraction using standard techniques, total hepatic cholesterol was determined by a cholesterol oxidase/esterase assay (Sigma Chemical Co., St Louis, Missouri, USA), as described previously, ${ }^{11}$ Unesterified cholesterol was measured by high performance liquid chromatography. ${ }^{29}$ The results are expressed in mg per gram of wet hepatic tissue.

\section{Immunoblotting of membrane transporters}

For western blots, $5 \mathrm{ml}$ of crude liver homogenate, each containing $2.9 \mathrm{mg} / \mathrm{ml}$ protein concentration (by Bradford ${ }^{28}$ assay with appropriate dilution), were mixed with $25 \mathrm{ml}$ of a commercially available sample buffer lithium dodecyl sulphate (Invitrogen Corporation, Carlsbad, California, USA), $60 \mathrm{ml} \mathrm{H}_{2} \mathrm{O}$, and $10 \mathrm{ml}$ of $100 \%$ 2-mercaptoethanol. Hence the protein concentrations in each sample were identical $(0.145 \mathrm{mg} / \mathrm{ml})$. Samples were denatured at $70^{\circ} \mathrm{C}$ for $10 \mathrm{~min}$ utes. Equal volumes of each sample were loaded onto a polyacrylamide gel, and electrophoresis was performed under reducing conditions. Proteins were then transferred to a nitrocellulose membrane and Ponceau $S$ staining was performed to validate equal protein transfers. Membranes were "blocked" using a mixture of milk, bovine serum albumin, and Tween 20. The primary antibodies were diluted in Tris buffered saline, pH 7.4, and detected by chemiluminescence using appropriate horseradish peroxidase conjugated secondary antibodies.

For $m d r 2$, the primary antibody was diluted at a ratio of 1:5000 in Tris buffered saline and incubated for two hours at room temperature. For bsep, the primary antibody was diluted at a ratio of 1:2000 and incubated for two hours at room temperature.

\section{Statistics}

Observed and expected intergroup frequencies of nonparametric data were compared using the $\chi^{2}$ method. Means of parametric data sets were compared using an independent sample Student's $t$ test; confidence intervals were $5 \%$ and $95 \%$. A p value of less than 0.05 was considered to be statistically significant. Correlations were determined using linear regression analysis. Values are expressed as mean (SEM).

\section{RESULTS}

In vitro HMGR activity, hepatic cholesterol stores, and plasma cholesterol levels

Mean weight gain over the experimental time frame (3.5 (1.5) g) and food consumption (3 (1) g/day) were similar in all groups. There was no mortality on diet alone or in the treatment groups, and all animals remained healthy. Figure 2 shows that the inhibitors downregulated the rate limiting enzyme in cholesterol biosynthesis in hepatic microsomal

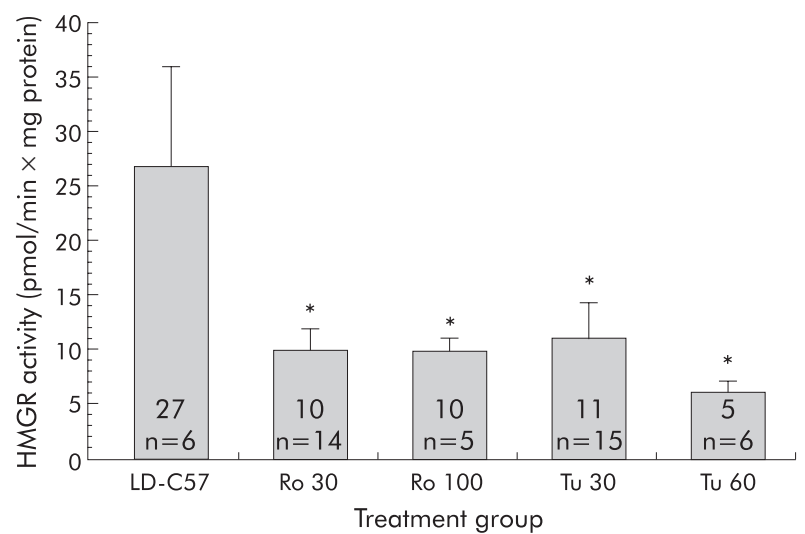

Figure 2 Hepatic microsomal 3-hydroxy-3-methylglutaryl-CoA reductase (HMGR) activities assayed ex vivo. Inhibitors significantly reduced activity uniformly compared with controls. In the case of the Ro 48-8071 agent, escalations in dose did not produce a proportionately greater degree of HMGR inhibition. In contrast, Tu 220860 achieved an approximately twofold reduction in HMGR activity compared with Tu 30, Ro 30, and Ro 100. Bars indicate means; ${ }^{*} p<0.05$ compared with controls (lithogenic diet (LD) alone). 
Table 1 Influence of inhibitors of cholesterol synthesis on hepatic cholesterol concentrations and esterified to free cholesterol ratio*

\begin{tabular}{llll}
\hline Treatment group & Total $\mathbf{~ ( m g / g ~ l i v e r ) ~}$ & Freet(mg/g liver) & Esterified to free ratio \\
\hline C57 LD & $13.1(0.9)$ & $0.86(0.21)$ & $15.6(3.1)$ \\
C57 simvastatin & $21.8(1.7) \ddagger$ & $1.18(0.24)$ & $22.2(4)$ \\
Ro 30 & $p=0.001$ & & \\
& $4.9(1.0) \ddagger$ & $0.51(0.04)$ & $8.0(1.3) \ddagger$ \\
Ro 60 & $p=0.03$ & & 0.02 \\
& $6.0(1.7) \ddagger$ & $0.76(0.22)$ & $7.7(2)$ \\
Ro 100 & $p=0.01$ & & $p=0.003$ \\
Tu 30 & $5.2(1.4) \ddagger$ & $0.52(0.07)$ & $9.0(2.2)$ \\
Tu 60 & $p=0.001$ & & $13.6(2.4)$ \\
\hline
\end{tabular}

*Analyses carried out at eight weeks on the lithogenic diet (LD).

†Hepatic cholesterol concentration assays were performed as described in the methods section. †Significantly different compared with the C57 controls fed the LD.

Hepatic cholesterol homeostasis was markedly changed by Ro and Tu. Striking reductions in total cholesterol were noted with all doses of Ro; much less marked reductions were effected by Tu. As expected, simvastatin caused hepatic cholesterol levels to increase. Levels of unesterified cholesterol remained substantially unchanged.

fractions, as inferred by the in vitro HMGR assay. As listed in table 1 , this effect was associated with a marked decrease in total hepatic cholesterol concentrations, albeit in a much more marked fashion with Ro $(41 \%$ and $76 \%$ of controls in the Ro and Tu groups, respectively). Neither Ro nor Tu however induced a significant decrease in hepatic free cholesterol concentration $(69 \%$ and $94 \%$ of controls, respectively), as evidenced by decreases in the esterified/free cholesterol ratios. Although a trend was apparent, no significant differences in plasma total cholesterol between inhibitor groups ( 152 (6) mg/dl (Ro) 158 (6) mg/dl (Tu)) and controls (165 (7) mg/dl) were found (56 days).

\section{Cholesterol gall stone prevalence rates}

Figure 3 shows that gall stone prevalence rates at 56 days decreased with increasing dose of Ro (respectively, 69\%, 57\%, and $17 \%$ at 30,60 , and $100 \mathrm{mg} / \mathrm{kg} / \mathrm{day})$. Gall stone prevalence rates also decreased (respectively, 29\% and 17\% with Tu 30

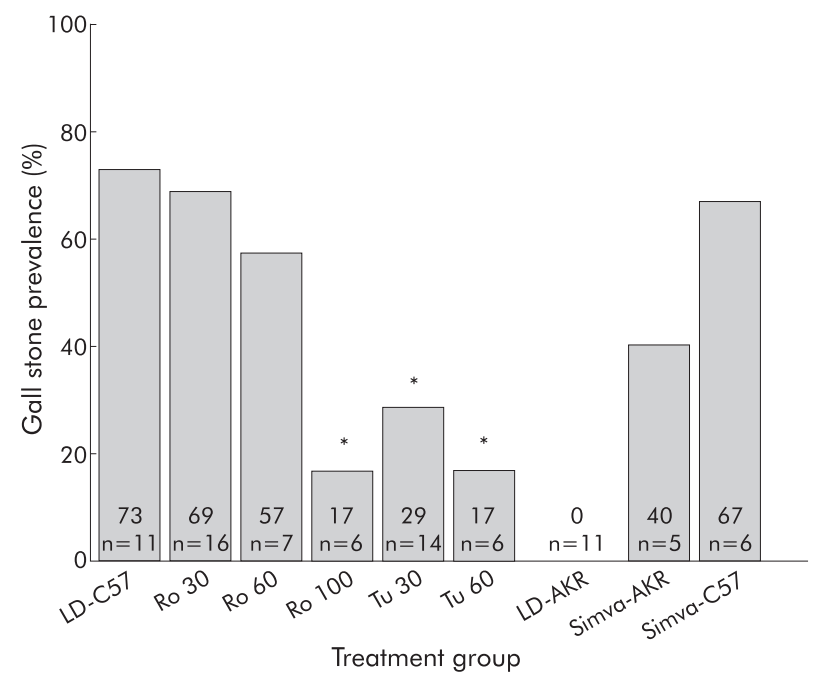

Figure 3 Gall stone prevalence rates expressed as a percentage in male $\mathrm{C} 57 \mathrm{~L} / \mathrm{J}$ mice fed a lithogenic diet (LD) with the addition of incremented doses of the Tu 2208 or Ro 48-8071 agents (see methods), and both C57 and AKR controls fed LD alone or LD plus simvastatin (simva). Statistically significant differences in prevalence rates were observed between Tu 30, Tu 60, and Ro 100 and both C57 control groups. There was no significant difference in gall stone prevalence between either AKR group. Bars and arithmetic values indicate prevalence rates; ${ }^{*} p<0.05$ compared with both controls. and $60 \mathrm{mg} / \mathrm{kg} /$ day). Stone prevalence in contemporaneous control animals fed LD alone was $73 \%$, with significant reductions in Ro 100 and Tu 30 and 60 treated animals. AKR mice fed LD alone exhibited a gall stone prevalence rate of $0 \%$ at the time point studied whereas those fed LD plus simva exhibited a prevalence rate of $40 \%$. This represents a statistical trend $\left(\mathrm{p}=0.10, \chi^{2}\right)$ (fig 3$)$.

\section{Biliary lipid secretion, lipid coupling, and cholesterol saturation indices}

Quantitation of biliary lipid secretion rates in hepatic bile employing an acute (one hour) fistula is shown in table 2 . Overall, volumes of secreted bile did not show any significant changes between groups. Cholesterol secretion rates were not significantly decreased in any group receiving the cholesterol synthesis inhibitors; on the contrary, there were statistically significant increases in cholesterol secretion rates in C57 Ro 30 and Ro 60 groups compared with controls (LD alone). In the case of AKR mice, no statistically significant changes were observed in Ro or simva fed animals. Bile salt secretion rates were unaffected by either drug at any dose level compared with control mice fed the LD diet alone. None the less, Ro treated mice exhibited significantly higher bile salt secretion rates than mice receiving Tu. However, with respect to biliary phosphatidylcholine (PC) secretion, both drugs resulted in significant increases in PC secretion rates compared with control mice. The response of LD diet fed AKR mice to Ro 100 was identical to that observed in the C57L strains with the same dose. Mice exhibited unchanged total bile salt secretion rates (314 (59) in Ro $100 v 381$ (153) in controls), cholesterol secretion rates (20 (3) in Ro $100 v 18$ (5) in controls, respectively) but significantly higher biliary PC outputs (58 (9) in Ro $100 v 42$ (10) in controls, respectively). This confirms that high Ro doses uncouple PC secretion from that of bile salt secretion, irrespective of the presence or absence of gall stone susceptible (Lith) alleles (table 2).

We verified the changes in phospholipid content of bile (as determined by the Bartlett method) by quantifying the choline moiety of phospholipids after choline oxidase hydrolysis. Our data (not shown) are consistent with our previous determination and establish that the increases in phospholipid secretory rates are not artefacts. In most samples, Bartlett and choline assays were within 10\% of each other; however, in one analysis of an Ro treated animal (at a dose of $30 \mathrm{mg} / \mathrm{kg} /$ day), the results were divergent. In this case, the Bartlett assay yielded an appreciably higher value for phospholipid secretion and employing TLC with 
Table 2 Biliary lipid secretion and cholesterol saturation indices

\begin{tabular}{|c|c|c|c|c|}
\hline Treatment group (No) & BSt & $\mathrm{PC} \dagger$ & Cholt & CSIł \\
\hline \multicolumn{5}{|l|}{ C57L-LD } \\
\hline & $657(62)$ & $64(10)$ & $19(4)$ & 106 (14) \\
\hline \multicolumn{5}{|l|}{ Ro 30} \\
\hline 6 & $824(79)$ & $138(6)^{*}$ & $58160^{*}$ & 149 (15) \\
\hline \multicolumn{5}{|l|}{ Ro 60} \\
\hline Ro 100 & $766(156)$ & $111(18)^{*}$ & $43(7)^{*}$ & $131(22)$ \\
\hline 4 & $692(29)$ & $94180^{*}$ & $26(2)$ & $80(5)$ \\
\hline \multicolumn{5}{|l|}{ Tu 60} \\
\hline 5 & $520(47)$ & $104(5)^{*}$ & $28(3)$ & $92(10)$ \\
\hline \multicolumn{5}{|l|}{ AKR-LD } \\
\hline 6 & 381 (153) & $42(10)$ & $18(5)$ & $79(8)$ \\
\hline \multicolumn{5}{|l|}{ AKR-simva } \\
\hline & $415(89)$ & $64(10)^{*}$ & $26(4)$ & 120 (1) \\
\hline \multicolumn{5}{|l|}{ C57-simva } \\
\hline & $480(48)$ & $67(9)$ & $18(2)$ & $88(16)$ \\
\hline \multicolumn{5}{|l|}{ AKR-Ro 100} \\
\hline 5 & $314(59)$ & $58(9)^{*}$ & $20(3)$ & $112(8)$ \\
\hline \multicolumn{5}{|c|}{$\begin{array}{l}\text { LD, lithogenic diet; BS, bile salt; PC, phosphatidylcholine (biliary phospholipid); Chol, cholesterol; CSI, cholesterol } \\
\text { saturation index. } \\
\text { †Values are expressed as nmol/min } \times 100 \mathrm{~g} \text { (body weight). } \\
\text { †Dimensionless values. } \\
\text { * } \mathrm{p}<0.05 \text { v controls. } \\
\mathrm{C} 57 \mathrm{~L} \text { mice fed Ro displayed higher BS secretion rates than controls whereas the single Tu fed group showed lower } \\
\text { levels. Higher Chol secretion rates were observed in Ro } 30 \text { and Ro } 60 \text { fed animals compared with controls. } \\
\text { Statistically significant elevations in biliary phospholipid secretion rates compared with controls were demonstrated } \\
\text { in all Ro and Tu drug groups in both C57L and AKR mice. Chol and BS secretion were not changed significantly in } \\
\text { AKR mice. CSI of murine hepatic bile in the fed state were calculated using critical tables }{ }^{26} \text { for taurocholate, the } \\
\text { principal murine bile salt in the LD. Chol desaturation of bile was evident in the Ro } 100 \text { and Tu } 60 \text { treatment groups } \\
\text { as well as in AKR mice on LD alone. All other groups displayed supersaturated and lithogenic bile, as evidenced by } \\
\text { the study. }\end{array}$} \\
\hline
\end{tabular}

appropriate standards, an unknown polyphosphated organic compound was present at the origin but its chemical nature was not pursued further. As expected from the aforementioned data, biliary lipid coupling ratios were modified by Ro and $\mathrm{Tu}$ administration. Cholesterol:bile salt ratios were significantly higher in the Ro $30(251 \%, p=0.004)$, Ro 60 $(212 \%, p=0.03)$, and Tu $60(192 \%, p=0.05)$ groups than in controls. Although the cholesterol:bile salt ratios did not differ in a statistically significant manner, the trend was towards higher values in the Ro 30 and Ro 60 groups. However, individual PC:bile salt ratios were all significantly increased compared with controls following Ro 30 (177\%, $\mathrm{p}=0.003)$, Ro $60(153 \%, \mathrm{p}=0.01)$, and Tu $(211 \%, \mathrm{p}=0.001)$ treatment. CSIs of hepatic bile (table 2) revealed values diminishing with escalating Ro dose, thereby coinciding with the cholesterol gall stone prevalence rates (fig 3). At the time of measurement in the morning, unsaturated bile was secreted only by Ro 100 and Tu 60 fed mice.

\section{Hepatic cholesterol concentration}

Hepatic cholesterol homeostasis was markedly changed by Ro and $\mathrm{Tu}$, as shown in table $\mathrm{l}$. Striking reductions in total cholesterol were noted with all doses of Ro; much less marked reductions were effected by Tu. As expected, simvastatin caused hepatic cholesterol levels to increase. Levels of unesterified cholesterol remained substantially unchanged (table 1).

\section{Hepatic expression of canalicular transporters}

Bar graphs quantifying expression of Mdr2 (ATP binding cassette $\mathrm{B} 4-\mathrm{ABCB} 4)$, the canalicular PC transmembrane translocator ( $\mathrm{PC}$ "flippase"), and bsep, the bile salt export pump (ABCB11), by immunoblotting are shown in fig 4. Ro but not Tu significantly increased $M d r 2$ expression compared with controls fed LD alone. However, bsep expression was unchanged between all drug groups and controls.

\section{DISCUSSION}

This study examined the in vivo effects of inhibitors of cholesterol synthesis, acting distally to oxidation of squalene (fig 1), in inbred mouse strains that possesses variant Lith alleles. $^{21}$ To allow the gall stone phenotype to become manifest, the model requires dietary administration of large amounts of cholesterol as well as cholic acid, ensuring a predominant change in the bile salt pool to that of sodium

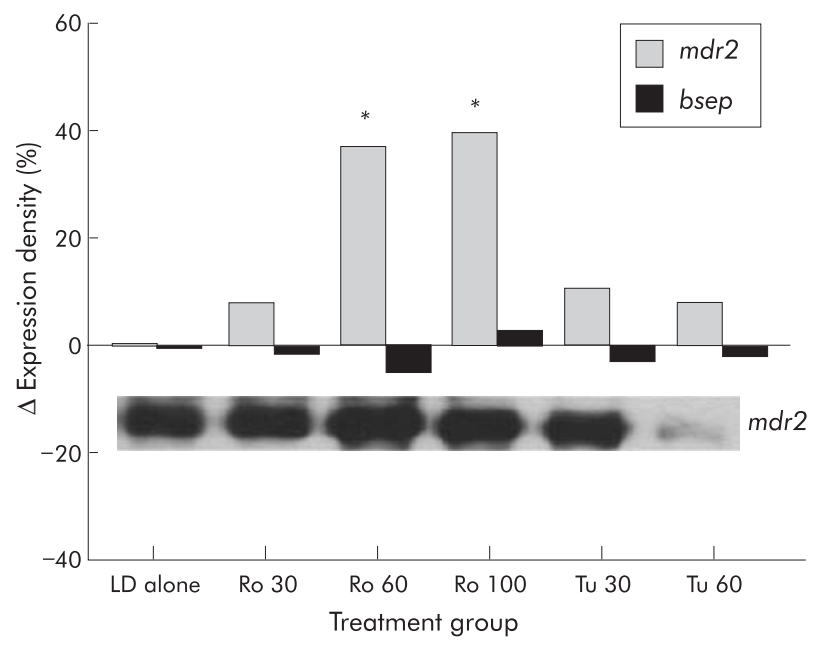

Figure 4 Results of digitally quantified multidrug resistance protein, isoform 2 (mdr2) and bile salt export protein (bsep) immnoblot expression in murine livers. In animals fed Ro 48-8071, mdr2 expression increased with escalating dose. In contrast, the results were variable in the case of Tu 2208. In both inhibitor groups, bsep expression did not change compared with controls (lithogenic diet (LD) alone). Histogram indicates means of $n=3-5$ provided as a percentage of changes in expression density compared with controls. Inset demonstrates a representative immunoblot for $m d r 2$, with labelling as per the $X$ axis. 
taurocholate. ${ }^{30}$ Not only does this increase the mass of cholesterol absorbed from the gut but it also promotes biliary secretion of cholesterol and facilitates a solid crystalline phase transition within gall bladder bile. ${ }^{30}$ Therefore, given this state of augmented mass movement of cholesterol from the gut to the liver and secretion into bile, any change in the rates of de novo cholesterol biosynthesis may be commensurately difficult to discern. However, they may be inferred from two measures; firstly, hepatic HMGR activity (reduced threefold in Ro 30, 100, and Tu 30 groups, and fivefold in the Tu 60 group); and secondly, hepatic cholesterol concentration (halved in Ro fed animals and reduced by one third in Tu 60), which is perhaps the best yardstick of cholesterol homeostasis in any of the experimental animals.

Although we do not know if circadian changes in biliary secretion occur in mice fed a lithogenic diet, the possibility of such circadian changes exists. However, to obviate any possibility that circadian factors might have interfered with our study, we administered the pharmacological agents admixed in the diet (thereby providing a sustained, usually nocturnal, intake) and secondly, by performing our surgical protocols at the same hour (and in random fashion) daily, we controlled for such effects.

We found that despite significantly reduced HMGR activities (fig 2) and hepatic cholesterol concentrations (table 1), neither Ro 48-8071 nor Tu 2208 decreased biliary cholesterol secretion, irrespective of the presence or absence of mutant Lith alleles. Clearly, neither agent directly inhibited HMGR (fig 1). However, both caused accumulation of cholesterol synthesis intermediaries to occur which are believed to directly or indirectly inhibit HMGR. ${ }^{31-33}$

Simvastatin associated upregulation of cholesterol synthesis increased gall stone prevalence rates in resistant AKR animals but not in the susceptible C57L strain. This is probably an effect of the variant Lith alleles, as our previous work has shown that LD causes downregulation of Hmgr in the AKR but not in the C57L strain. ${ }^{11}$

Despite evidence ${ }^{34}$ that de novo synthesised cholesterol in rodents may make a marked contribution to bile salt synthesis and bile secretion, neither of the inhibitors tested exhibited significant effects on bile salt secretion. On the contrary, a tendency towards increased bile salt secretion was observed in groups receiving Ro (table 2). LD is a potent downregulator of Cyp7al, ${ }^{11}$ as previous work ${ }^{11}$ has shown that the latter is suppressed by cholic acid present in the LD and by deoxycholic acid, its bacterial metabolite, via the farnesoid $X$ receptor. ${ }^{35}$ To account for increased bile salt secretion, it is also tempting to speculate that Ro 48-8071 may increase hepatic uptake and secretion of cholic acid metabolites. Although we did not find increased hepatic bsep expression, it is possible that functional upregulation of bsep may occur principally by recruitment from a reservoir pool in the subapical domain rather than by increased transcription. $^{36}$

Although secretion rates of biliary cholesterol either remained the same or increased, Tu and high dose Ro significantly decreased gall stone prevalence rates in C57L mice (fig 3). This occurred as a consequence of marked increases in biliary phospholipid secretion, which increased the solubility of cholesterol in bile. ${ }^{26}$

It should be noted that CSIs of gall bladder bile are always less than those of hepatic bile, and so a supersaturated hepatic bile could be accompanied by unsaturated gall bladder bile. However, if hepatic bile is unsaturated, then gall bladder bile must by necessity also be unsaturated. ${ }^{30}$

Increased PC secretion has also been reported in statin treated rodents but this is offset by an accompanying increase in biliary cholesterol secretion. In the case of postHMGR inhibitors, the metabolic fate of the accumulated oxidosqualene intermediaries is not well known. If metabolised by glucuronidation and secreted subsequently into bile, the observed secretion rates of biliary lipids could have been influenced. In fact, it is believed ${ }^{32}$ that further oxidation occurs before slow elimination, as opposed to glucuronidation (Morand OH. Pharmacokinetics of Ro 48-0871. Personal communication, 2000). Notwithstanding, after phase II transformations, drugs that are secreted by MRP2, usually decrease biliary PC secretion, not augment it. ${ }^{14}{ }^{17}{ }^{37}$ Hooiveld and colleagues ${ }^{14}$ have speculated that statin induced intracellular depletion of cholesterol may cause increased expression of the transcription factor sterol regulatory element binding protein (SREBP), which is controlled by oxysterols and isoprenoids. ${ }^{12}{ }^{13}$ Transcription of Mdr2 (the transmembrane phosphatidylcholine translocase) is postulated to be upregulated through analogous, but yet uncharacterised, mechanisms and has also been demonstrated with squalene synthase inhibitors. ${ }^{38}$ We have found the same effects in the present work, particularly with the Ro inhibitor of oxidosqualene-lanosterol cyclase. The greater efficiency of Ro, as compared with $\mathrm{Tu}$, in decreasing hepatic cholesterol esters and to some extent free hepatic cholesterol (table 1) and in increasing $M d r 2$ expression (fig 4), gives further weight to the likely role of SREBP signalling pathways in its pharmacological actions. It is tempting therefore to suggest that the key regulatory sterol in $M d r 2$ transcription is formed distally to cyclisation of 2,3-oxidosqualene (fig 1).

If the principal findings of the work (that is, that of agent induced desaturation of bile by augmented biliary phospholipid secretion) can be extrapolated cautiously to humans, it is logical to speculate that the ability of Ro 48-8071 to induce secretion of both PC and to a lesser extent bile salts may have important clinical implications. This is not only for gall stone chemoprevention and dissolution, but also for the possible therapy of cholestatic liver diseases. Increased fibrosis was noted as an important phenotype in the $M d r 2^{--/}$mouse which worsened with administration of hydrophobic bile acids and was ameliorated with UDCA, emphasising the cytoprotective property of biliary PC and hydrophilic bile acids. $^{39} 40$ We speculate that inhibitors of both squalene epoxidase and squalene-lanosterol cyclase may play an additional role, not only as antihypercholesterolaemic agents $^{15}{ }^{19}$ but also as agents that upregulate biliary phospholipid secretion. If this can be proved to occur in humans, they may also offer a means of both gall stone chemoprevention and a new therapeutic option for a variety of cholestatic diseases where secretion of biliary phospholipid is compromised.

\section{ACKNOWLEDGEMENTS}

The authors express their gratitude to Ms Monika Leonard, Brigham and Women's Hospital, Boston, MA, USA, and Mr Harry Whitmore, the Jackson Laboratory, Bar Harbor, ME, USA, for their outstanding technical assistance. Dr Olivier Morand (F Hoffmann-La Roche, Basel, Switzerland) is thanked particularly, not only for his generous gift of Ro 48-8071, but also for his insightful comments during the course of this work and his critical reading of the manuscript.

Supported in part by AASLD/Schering Advanced Hepatology Fellowship (GC) and Institutional Training NIDDK grant DK 753315 (GC), Research and Center NIDDK grants DK 36588, 52911, 38454 (MCC), and DK51568 (BP) from the National Institutes of Health (US Public Health Service).

\section{Authors' affiliations}

G A Clarke, G Bouchard, M C Carey, Department of Medicine, Harvard Medical School, Division of Gastroenterology, Brigham and Women's Hospital and Harvard Digestive Diseases Center, Boston, MA 02115, USA

B Paigen, The Jackson Laboratory, Bar Harbor, ME 04609, USA 


\section{REFERENCES}

1 Cooper AD. Hepatic uptake of chylomicron remnants. J Lipid Res 2001;38:2173-92.

2 Joyce C, Skinner K, Anderson RA, et al. Acyl-coenzyme A: cholesterol acyl transferase 2. Curr Opin Lipidol 1999;10:89-95.

3 Kang S, Davis RA. Cholesterol and hepatic lipoprotein assembly and secretion. Biochim Biophys Acta 2000;1529:223-30.

4 Amigo L, Quinones V, Mardones $P$, et al. Impaired biliary lipid secretion and decreased gallstone formation in alipoprotein E-deficient mice fed a highcholesterol diet. Gastroenterology 2000;1 18:772-9.

5 Scheibner J, Fuchs M, Hormann E, et al. Biliary cholesterol secretion and bile acid formation in the hamster: the role of newly synthesized cholesterol. J Lipid Res 1994; 35:690-7

6 Turley SD, Spady DK, Dietschy JM. Alteration of the degree of biliary cholesterol saturation in the hamster and rat by manipulation of the pools of preformed and newly synthesized cholesterol. Gastroenterology 1983:84:253-64

7 Wang DQ, Lammert F, Paigen B, et al. Phenotypic characterization of lith genes that determine susceptibility to cholesterol cholelithiasis in inbred mice. Pathophysiology of biliary lipid secretion. J Lipid Res 1999;40:2066-79.

8 Wang DQ, Paigen B, Carey MC. Phenotypic characterization of Lith genes that determine susceptibility to cholesterol cholelithiasis in inbred mice: physical-chemistry of gallbladder bile. J Lipid Res 1997;38:1395-41 1 .

9 Fuchs $M$, Ivandic B, Muller O, et al. Biliary cholesterol hypersecretion in gallstone-susceptible mice is associated with hepatic up-regulation of the high density lipoprotein receptor, SR-B1. Hepatology 2000;33:1451-9.

10 Fuchs $M$, Lammert $F$, Wang DQ, et al. Sterol carrier protein 2 participates in hypersecretion of biliary cholesterol during gallstone formation in genetically gallstone-susceptible mice. Biochem J 1998;336:33-7.

11 Lammert $F$, Wang DQ, Paigen B, et al. Phenotypic characterization of Lith genes that determine susceptibility to cholesterol cholelithiasis in inbred mice: integrated activities of hepatic lipid regulatory enzymes. J Lipid Res 1999;40:2080-90

12 Edwards PA, Tabor D, Kast HR, et al. Regulation of gene expression by SREBP and SCAP. Biochim Biophys Acta 2000;1529:103-13.

13 Edwards PA, Ericsson J. Sterols and isoprenoids: signaling molecules derived from the cholesterol biosynthetic pathway. Annu Rev Biochem 1999;68: 157-85

14 Hooiveld GJ, Vos TA, Scheffer GL, et al. 3-Hydroxy-3-methylglutarylcoenzyme A reductase inhibitors (statins) induce hepatic expression of the phospholipid translocase mdr2 in rats. Gastroenterology 1999;117:678-87.

15 Morand OH, Aebi JD, Dehmlow H, et al. Ro 48-8.071, a new 2,3oxidosqualene:lanosterol cyclase inhibitor lowering plasma cholesterol in hamsters, squirrel monkeys, and minipigs: comparison to simvastatin. J Lipid Res 1997:38:373-90.

16 Fujioka T, Nara F, Tsujita Y, et al. The mechanism of lack of hypocholesterolemic effects of pravastatin sodium, a 3-hydroxy-3 methylglutaryl coenzyme A reductase inhibitor, in rats. Biochim Biophys Acta 1995; 1254:7-12.

17 Linscheer WG, Atreyee B, Uma KM, et al. Lovastatin induces synthesis of cholesterol, which acts as a secretagogue of biliary phospholipids in rats. Am J Physiol 1995;268:G242-50.

18 Koide K, Hayashi K, Horiuchi I, et al. Effect of CS-514, a competitive inhibitor of hydroxymethylglutaryl coenzyme A reductase, on cholesterol gallstone formation in hamsters. Biochim Biophys Acta 1989;1005:65-71.

19 Matzno S, Yamauchi T, Gohda M, et al. Inhibition of cholesterol biosynthesis by squalene epoxidase inhibitor avoids apoptotic cell death in $L 6$ myoblasts. $J$ Lipid Res 1997:38:1639-48.

20 Gerloff T, Stieger B, Hagenbuch B, et al. The sister of P-glycoprotein represents the canalicular bile salt export pump of mammalian liver. $J$ Biol Chem 1998;273:10046-50.
21 Khanuja B, Cheah YC, Hunt $M$, et al. Lith 1, a major gene affecting cholesterol gallstone formation among inbred strains of mice. Proc Natl Acad Sci U S A 1995;92:7729-33.

22 Allain CC, Poon LS, Chan CS, et al. Enzymatic determination of total serum cholesterol. Clin Chem 1974;20:470-5.

23 Bartlett GR. Phosphorus assay in column chromatography. J Biol Chem 1959;234:466-8

24 Turley SD, Dietschy JM. Re-evaluation of the 3 alpha-hydroxysteroid dehydrogenase assay for total bile acids in bile. J Lipid Res 1978;19:924-8.

25 Rossi SS, Converse JL, Hofmann AF. High pressure liquid chromatographic analysis of conjugated bile acids in human bile: simultaneous resolution of sulfated and unsulfated lithocholyl amidates and the common conjugated bile acids. J Lipid Res 1987;28:589-95.

26 Carey MC. Critical tables for calculating the cholesterol saturation of native bile. J Lipid Res 1978; 19:945-55.

27 Doerner KC, Gurley EC, Vlahcevic ZR, et al. Regulation of cholesterol 7 alphahydroxylase expression by sterols in primary rat hepatocyte cultures. J Lipid Res 1995;36:1 168-77.

28 Bradford MM. A rapid and sensitive method for the quantitation of microgram quantities of protein utilizing the principle of protein-dye binding. Anal Biochem 1976;72:248-54.

29 Vercaemst R, Union A, Rosseneu M, et al. Quantitation of plasma free cholesterol and cholesteryl esters by high performance liquid chromatography. Study of a normal population. Atherosclerosis 1989:78:245-50.

30 Wang DQ, Lammert F, Cohen DE, et al. Cholic acid aids absorption, biliary secretion, and phase transitions of cholesterol in murine cholelithogenesis. Am J Physiol 1999;276:G751-60.

31 Panini SR, Delate TA, Sinensky M. Post-transcriptional regulation of 3hydroxy-3-methylglutaryl coenzyme A reductase by $24(\mathrm{~S}), 25$-oxidolanosterol post-transcriptional regulation of 3-hydroxy-3-methylglutaryl coenzyme A reductase by 24(S),25-oxidolanosterol. J Biol Chem 1992;267:12647-54.

32 Spencer TA, Gayen AK, Phirwa S, et al. 24(S), 25-Epoxycholesterol. 24(S), 25Epoxycholesterol. Evidence consistent with a role in the regulation of hepatic cholesterogenesis. J Biol Chem 1985;260:13391-4.

33 Taylor FR, Kandutsch AA, Gayen AK, et al. 24,25-Epoxysterol metabolism in cultured mammalian cells and repression of 3-hydroxy-3-methylglutaryl-CoA reductase 24,25-epoxysterol metabolism in cultured mammalian cells and repression of 3-hydroxy-3-methylglutaryl-CoA reductase. J Biol Chem 1986;261:15039-44

34 Vlahcevic ZR, Pandak WM, Hylemon PB et al. Role of newly synthesized cholesterol or its metabolites on the regulation of bile acid biosynthesis after short-term biliary diversion in the rat. Hepatology 1993; 18:660-8.

35 Makishima M, Okamoto AY, Repa JJ, et al. Identification of a nuclear receptor for bile acids. Science 1999;284:1362-5.

36 Schmitt M, Kubitz R, Lizun S, et al. Regulation of the dynamic localization of the rat Bsep gene-encoded bile salt export pump by anisoosmolarity. Hepatology 2001;33:509-18.

37 Gerk PM, Vore M. Regulation of expression of the multidrug resistanceassociated protein 2 (MRP2) and its role in drug disposition. J Pharmacol Exp Ther 2002;302:407-15.

38 Gupta S, Todd Stravitz R, Pandak WM, et al. Regulation of multidrug resistance $2 \mathrm{P}$-glycoprotein expression by bile salts in rats and in primary cultures of rat hepatocytes. Hepatology 2000;32:341-7.

39 Oude Elferink RP, Ottenhoff R, van Wijland $M$, et al. Regulation of biliary lipid secretion by mdr2 P-glycoprotein in the mouse. $J$ Clin Invest 1995:95:31-8

40 Smit JJ, Schinkel AH, Oude Elferink RP, et al. Homozygous disruption of the murine mdr2 P-glycoprotein gene leads to a complete absence of phospholipid from bile and to liver disease. Cell 1993;75:451-62. 